\title{
COVID-19 Impact on Ride-hailing: The Chicago Case Study
}

\author{
Jianhe Du ${ }^{1}$ (D), Hesham A. Rakha ${ }^{2}$ (D) ${ }^{a}$ \\ ${ }^{1}$ Virginia Tech Transportation Institute, Virginia Polytechnic Institute and State University (VA), ${ }^{2}$ Charles E. Via, Jr. Dept. of Civil and Environmental Engineering, \\ Virginia Polytechnic Institute and State University (VA) \\ Keywords: covid-19, uber, ride-hailing, ridesharing
}

\section{Findings}

A dramatic traffic volume reduction has been observed worldwide and across the United States since the onset of the COVID-19 pandemic. This event provides us with a unique opportunity to investigate the changes in ride-hailing travel prior and during the pandemic and the resulting significant drop in economic activities associated with the pandemic. In this paper, we collected and analyzed trip-bytrip ride-hailing data from the City of Chicago over 6 months (January to June) in 2019 and 2020. The results from Chicago show a significantly greater decrease in the number of ride-hailing trips compared to those using personal vehicles during the pandemic. In most cases, the average travel distances lengthened while the average travel times shortened. Pooled trips decreased significantly (71\%) in early March and disappeared completely on March 17 when Uber and Lyft suspended trip pooling. Short trips that used to occur within the same census tracts decreased remarkably. To our knowledge this is the first effort to analyze ride-hailing data as related to COVID-19. We hope that this effort serves as a first step towards conducting other studies in other cities.

\section{RESEARCH QUESTION}

The research question we were interested in answering in this paper is what changes COVID-19, and the dramatic drop in human activities associated with COVID-19, have had on the ride-hailing industry. In this paper, we investigate the changes in ride-hailing travel with regard to the number of total trips, number of pooled trips, number of single trips, travel frequency, trip travel times, and trip distances on weekdays and weekends, as well as the variations in longer (travels across census tracts) and shorter (internal travels within a census tract) trips during the COVID-19 pandemic. While our analysis is specific to Chicago, we anticipate that these findings could be reflective of other North American cities.

\section{METHODS AND DATA}

The City of Chicago began collecting trip data from Transportation Network Providers (TNPs) comprising Uber and Lyft in November 2018 (Chicago Data Portal; https://data.cityofchicago.org/). The trip database has fields that include pick-up and drop off information (time, date, location, community area); trip duration (seconds) and distance; fare; shared trip (true/false), and

\footnotetext{
a Corresponding Author: hrakha@vt.edu Tel: (540) 231-1505 Virginia Tech Transportation Institute, 3500 Transportation Research Plaza Blacksburg, VA, 24060; United States
} 
Table 1. Traffic volume changes by destination (data retrieved up to April 10, 2020).

\begin{tabular}{l|ccccc}
\hline & Retail \& Recreation & Grocery \& Pharmacy & Parks & Transit Stations & Work \\
\hline \multirow{2}{*}{ Virginia } & $-44 \%$ & $-18 \%$ & $22 \%$ & $-56 \%$ & $-39 \%$ \\
California & $-53 \%$ & $-27 \%$ & $-61 \%$ & $-59 \%$ & $-42 \%$ \\
Nationwide & $-49 \%$ & $-20 \%$ & $-20 \%$ & $-54 \%$ & $-40 \%$ \\
\hline
\end{tabular}

number of passengers. This is the most detailed ride-hailing trip database that we are aware of and provides enough trip information to address the research question.

As illustrated in Table 1, on average the traffic volume in the United States decreased somewhere between $45 \%$ and $55 \%$ (the number varies depending on how badly the COVID-19 hit an area). We compared a total of 82 million ridehailing trips covering 52 weeks from January through June in 2019 and 2020. As seen in Table 2 and Figure 1, single ride-hailing trips (non-shared trips) were on average 17\% higher in 2020 in the first nine weeks (two months) of the year and then dropped significantly in March 2020. ${ }^{1}$ Beginning from the week of March 11, single ride-hailing trips decreased by more than $75 \%$ and this trend continued until the end of $\mathrm{May}^{2}$. This reduction amount is much larger than the general traffic volume drops in the United States. Subsequently, the number of single ride-hailing trips started increasing slowly in June 2020 after the stay-at-home order ended although they were still 55\% lower than that for the same period in 2019. In the case of pooled trips (trips that were labeled as shared and had more than a single passenger ${ }^{3}$ ), the numbers for 2020 were lower by $50 \%$ over the first eight weeks, indicating a drop in pooled trips unrelated to COVID- 19 . Pooled trips then dropped abruptly by $71 \%$ in early March 2020 with the pooled trips reaching zero on March 17 when TNPs suspended their shared services (Buzzfeednews 2020).

Figure 1 illustrates the weekly fluctuation in ride-hailing trips over the first six months of each year. Week 10 represents the week that the first COVID-19 case was reported in Chicago; week 12 was the week when TNPs suspended ride pooling services; week 13 was the week the stay-at-home order was initiated; and week 23 was when the stay-at-home order ended. The figure clearly shows that the number of trips decreased significantly after the onset of the COVID-19 virus and the associated stay-at-home order that was issued.

\footnotetext{
1 The stay at home order was issued on March 26, 2020 and remained until June 3, 2020. This order closed all of Chicago parks, beaches, and trails on and adjacent to the Lakefront, the Chicago Riverwalk, and the Bloomingdale Trail (the 606). People were allowed go for walks or jogs but had to maintain at least six feet distance from others. Sports events were canceled. Any get-togethers of more than ten people in public or at home were also not allowed. The Police Department helped in making sure that people were following the rules.

2 The first COVID-19 case in the US was reported on January 21, 2020. This case was reported in Washington State. The first COVID-19 case reported in Chicago was reported on March 3, 2020.

$399.9 \%$ of trips that had more than a single passenger were labeled as shared in the database.
} 
Table 2. Weekly Variations for 2019 and 2020

\begin{tabular}{|c|c|c|c|c|c|c|}
\hline \multirow{2}{*}{ Week } & \multicolumn{2}{|c|}{ Date on Start of Week } & \multicolumn{2}{|c|}{ Number of Trips (Single/Pooled, Thousands) } & \multicolumn{2}{|c|}{ Changes (\%) } \\
\hline & 2019 & 2020 & 2019 & 2020 & Single & Pooled \\
\hline 1 & $1 / 1 / 2019$ & $1 / 1 / 2020$ & $1384 / 321$ & $1605 / 165$ & $16 \%$ & $-49 \%$ \\
\hline 2 & $1 / 8 / 2019$ & $1 / 8 / 2020$ & $1523 / 362$ & $1793 / 204$ & $18 \%$ & $-44 \%$ \\
\hline 3 & $1 / 15 / 2019$ & $1 / 15 / 2020$ & $1570 / 383$ & $1895 / 221$ & $21 \%$ & $-42 \%$ \\
\hline 4 & $1 / 22 / 2019$ & $1 / 22 / 2020$ & $1621 / 427$ & $1833 / 210$ & $13 \%$ & $-51 \%$ \\
\hline 5 & $1 / 29 / 2019$ & $1 / 29 / 2020$ & $1501 / 417$ & $1813 / 200$ & $21 \%$ & $-52 \%$ \\
\hline 6 & $2 / 5 / 2019$ & $2 / 5 / 2020$ & $1628 / 414$ & $1870 / 196$ & $15 \%$ & $-53 \%$ \\
\hline 7 & 2/12/2019 & $2 / 12 / 2020$ & $1668 / 415$ & $2117 / 217$ & $27 \%$ & $-48 \%$ \\
\hline 8 & 2/19/2019 & $2 / 19 / 2020$ & $1724 / 405$ & $1963 / 177$ & $14 \%$ & $-56 \%$ \\
\hline 9 & $2 / 26 / 2019$ & $2 / 26 / 2020$ & $1796 / 432$ & $1985 / 200$ & $11 \%$ & $-54 \%$ \\
\hline $10^{*}$ & $3 / 5 / 2019$ & $3 / 4 / 2020$ & $1764 / 438$ & $1866 / 187$ & $6 \%$ & $-57 \%$ \\
\hline 11 & $3 / 12 / 2019$ & $3 / 11 / 2020$ & $1866 / 437$ & $1494 / 128$ & $-20 \%$ & $-71 \%$ \\
\hline 12 & $3 / 19 / 2019$ & $3 / 18 / 2020$ & $1643 / 385$ & $491 / 0$ & $-70 \%$ & $-100 \%$ \\
\hline $13^{* *}$ & $3 / 26 / 2019$ & $3 / 25 / 2020$ & $1462 / 315$ & $357 / 0$ & $-76 \%$ & $-100 \%$ \\
\hline 14 & $4 / 2 / 2019$ & $4 / 1 / 2020$ & $1678 / 368$ & $338 / 0$ & $-80 \%$ & $-100 \%$ \\
\hline 15 & 4/9/2019 & $4 / 8 / 2020$ & $1739 / 368$ & $337 / 0$ & $-81 \%$ & $-100 \%$ \\
\hline 16 & $4 / 16 / 2019$ & $4 / 15 / 2020$ & $1581 / 331$ & $381 / 0$ & $-76 \%$ & $-100 \%$ \\
\hline 17 & 4/23/2019 & $4 / 22 / 2020$ & $1704 / 354$ & $371 / 0$ & $-78 \%$ & $-100 \%$ \\
\hline 18 & $4 / 30 / 2019$ & $4 / 29 / 2020$ & $1779 / 360$ & $414 / 0$ & $-77 \%$ & $-100 \%$ \\
\hline 19 & $5 / 7 / 2019$ & $5 / 6 / 2020$ & $1691 / 341$ & $413 / 0$ & $-76 \%$ & $-100 \%$ \\
\hline 20 & $5 / 14 / 2019$ & $5 / 13 / 2020$ & $1754 / 329$ & $425 / 0$ & $-76 \%$ & $-100 \%$ \\
\hline 21 & $5 / 21 / 2019$ & $5 / 20 / 2020$ & $1709 / 306$ & $444 / 0$ & $-74 \%$ & $-100 \%$ \\
\hline 22 & $5 / 28 / 2019$ & $5 / 27 / 2020$ & $1696 / 314$ & $444 / 0$ & $-74 \%$ & $-100 \%$ \\
\hline $23^{* * *}$ & 6/4/2019 & $6 / 3 / 2020$ & $1742 / 320$ & $526 / 0$ & $-70 \%$ & $-100 \%$ \\
\hline 24 & 6/11/2019 & $6 / 10 / 2020$ & $1762 / 320$ & $627 / 0$ & $-64 \%$ & $-100 \%$ \\
\hline 25 & $6 / 18 / 2019$ & $6 / 17 / 2020$ & $1813 / 254$ & $674 / 0$ & $-63 \%$ & $-100 \%$ \\
\hline 26 & $6 / 25 / 2019$ & $6 / 24 / 2020$ & $1608 / 191$ & $723 / 0$ & $-55 \%$ & $-100 \%$ \\
\hline
\end{tabular}

${ }^{*}$ First COVID-19 case reported. ${ }^{* *}$ Stay-at-home order starts. ${ }^{* * *}$ Stay-at-home order ends.

We then extracted the average trip travel times and distances of ride-hailing trips. Since pooled trips were not available for 2020, we only analyzed the single passenger trips for weekdays and weekends. As illustrated in Figure 2, single traveler trips traveled longer distances but had shorter travel times, revealing a less congested transportation system during weekdays. On weekends, single passenger trips showed a similar trend to that on weekdays, but the changes were on a larger scale. On average, over the weeks starting from March 4, 2020 (first COVID-19 case reported in Chicago) to the end of June, single passenger trips were $14 \%$ longer distance-wise, but $12 \%$ shorter time-wise on weekdays. On weekends, single passenger trips were $23 \%$ longer in distance and $6 \%$ shorter in time.

One argument about ride-hailing imposing extra burden to our traffic system is that it induces trips that would not have taken place were ride-hailing not available. These induced trips can either be trips made by travelers who are not capable of driving or can be short trips that used to be made via walking or 


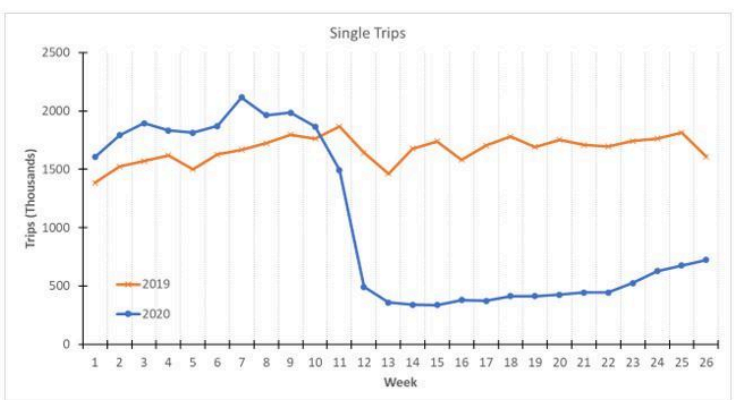

(a) Single Passenger Trips (Non-pooled trips)

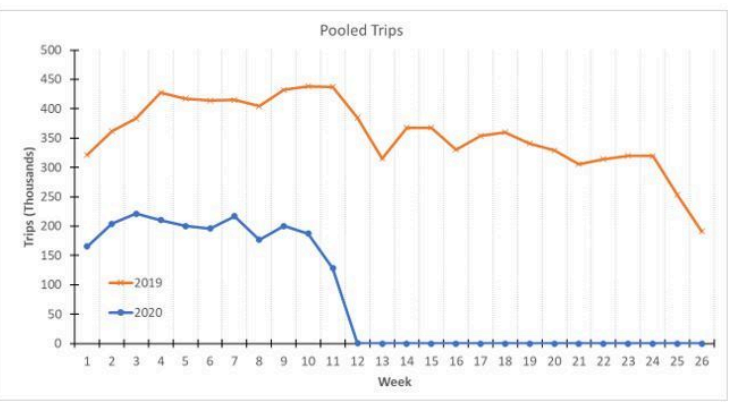

(b) Pooled Trips
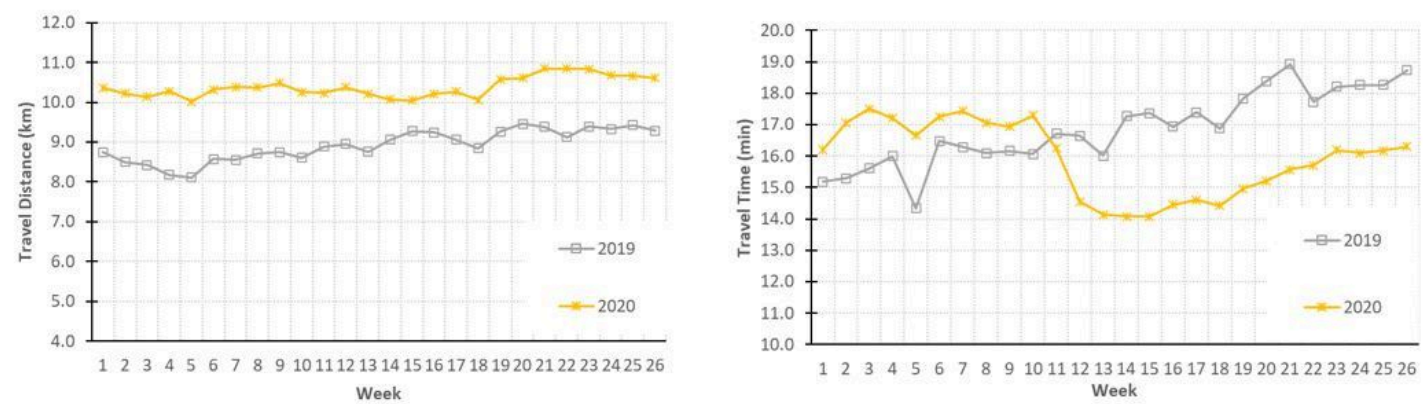

(a) Weekday Weekly Average
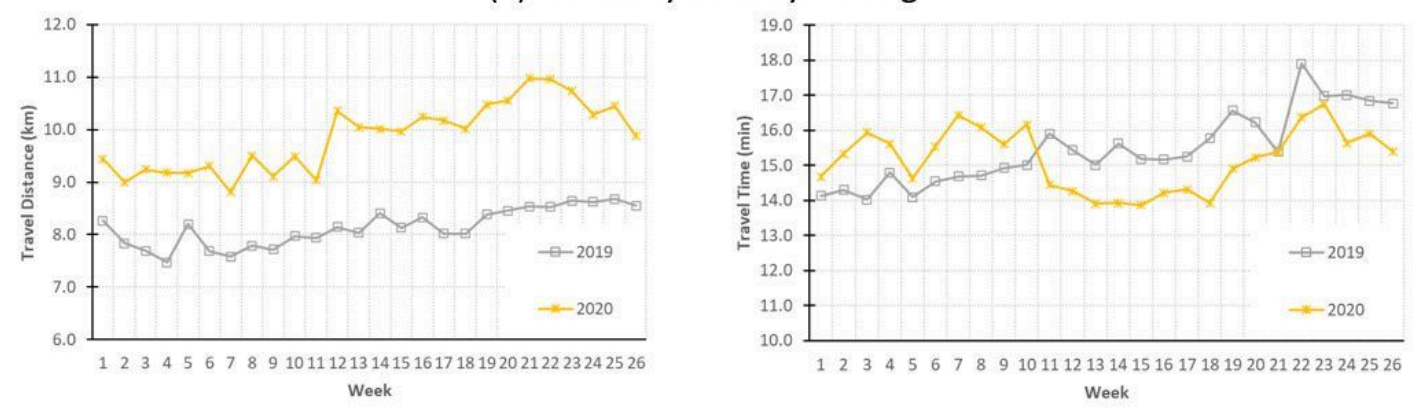

(b) Weekend Weekly Average

Figure 2. Travel Distances and Travel Times for Weekdays and Weekends

biking. The Chicago database provided us with a unique subset of ride-hailing trips: inter-census-tract trips where the origin and destination of a trip were within the same census tract. A close examination of these trips showed that changes in these short trips were more compatible with the amount of change 
Table 3. Internal Census-Tract Trips

\begin{tabular}{l|c|c|c|c|c|c}
\hline Month & $\begin{array}{c}2019 \\
\text { Trips }\end{array}$ & $\begin{array}{c}2020 \\
\text { Trips }\end{array}$ & Changes & $\begin{array}{c}2019 \text { Internal Tract } \\
\text { Trips }\end{array}$ & $\begin{array}{c}2020 \text { Internal Tract } \\
\text { Trips }\end{array}$ & Changes \\
\hline Jan & 368,329 & 345,299 & $-6.30 \%$ & 795 & 800 & $0.60 \%$ \\
Feb & 364,931 & 345,664 & $-5.30 \%$ & 814 & 803 & $-0.25 \%$ \\
Mar & 394,258 & 288,094 & $-26.90 \%$ & 829 & 771 & $-7.00 \%$ \\
April & 330,731 & 192,657 & $-41.70 \%$ & 782 & 446 & $-43.00 \%$ \\
May & 335,061 & 203,329 & $-39.30 \%$ & 792 & 506 & $-36.10 \%$ \\
June & 312,354 & 203,256 & $-34.90 \%$ & 784 & 602 & $-23.20 \%$ \\
\hline
\end{tabular}

Table 4. Changes in Internal and External Travels in Time (minutes) and Distance (KM)

\begin{tabular}{|c|c|c|c|c|c|c|c|}
\hline Level & Month & $\begin{array}{r}2019 \text { TT } \\
\text { (Min) }\end{array}$ & $\begin{array}{r}2020 \mathrm{TT} \\
\text { (Min) }\end{array}$ & $\begin{array}{r}\text { Change (\%) (T- } \\
\text { test Prob) }\end{array}$ & $\begin{array}{r}2019 \text { Dist } \\
\text { (KM) }\end{array}$ & $\begin{array}{r}2020 \text { Dist } \\
(\mathrm{KM})\end{array}$ & $\begin{array}{r}\text { Change (\%) (T- } \\
\text { test Prob) }\end{array}$ \\
\hline \multirow{6}{*}{ Internal } & Jan & 6.9 & 7.1 & $3.0(0)$ & 2.4 & 2.72 & $16.2(0)$ \\
\hline & Feb & 7.4 & 7.3 & $-1.1(2 e-5)$ & 2.56 & 2.72 & $7.9(0)$ \\
\hline & Mar & 7.5 & 7.4 & $-0.6(0.047)$ & 2.72 & 2.88 & $5.9(0)$ \\
\hline & Apr & 7.0 & 7.9 & $12.4(0)$ & 2.4 & 3.04 & $26.7(0)$ \\
\hline & May & 7.2 & 8.3 & $14.0(0)$ & 2.4 & 3.2 & $33.3(0)$ \\
\hline & June & 7.2 & 8.5 & $17.3(0)$ & 2.56 & 3.2 & $25.0(0)$ \\
\hline \multirow{6}{*}{ External } & Jan & 16.6 & 17.3 & $4.0(0)$ & 8.64 & 10.56 & $21.0(0)$ \\
\hline & Feb & 17.3 & 17.4 & $1.0(0)$ & 8.8 & 10.4 & $19.3(0)$ \\
\hline & Mar & 17.4 & 16.9 & $-2.8(0)$ & 8.96 & 10.72 & $19.6(0)$ \\
\hline & Apr & 17.9 & 15.2 & $-15.0(0)$ & 9.28 & 11.2 & $20.7(0)$ \\
\hline & May & 18.7 & 16.2 & $-13.1(0)$ & 9.44 & 11.68 & $23.7(0)$ \\
\hline & June & 18.7 & 16.8 & $-10.3(0)$ & 9.44 & 11.2 & $18.6(0)$ \\
\hline
\end{tabular}

in overall traffic volume (Table 2). The percentage of inter-tract travel decrease was about $40 \%$ in April and May, in accordance with the overall decrease in traffic (Du and Rakha 2020). In census tracts where these short internal tract trips occurred, we can see a sharp drop in April, when $43 \%$ of tracts had no internal trips at all.

We further examined the travel distances and travel times for both internal census tract travels and trips that covered more than one census tract (i.e., external). As shown in Table 3 , there was an increase in travel distances for both internal and external travels. The incremental change in travel distance for internal trips was more significant, especially during the months of April through June. The travel times for internal trips increased as well, indicating significantly longer internal trips. Even with the offset of less congested travel conditions, there were still significant increases in travel time for these internal trips. External trip distances also increased. Due to the lower traffic volume in the network, travel times for external tract trips decreased. All the results are statistically significant with $p$-values less than 0.05 considering a two-sample $T$ test. 


\section{FINDINGS}

In this work, we extracted a large trip-by-trip ride-hailing dataset for the City of Chicago including rideshare and single passenger trips from January through June in 2019 and 2020. These trips included trips in Chicago prior and after the onset of the COVID-19 pandemic. The data were analyzed to compare ride-hailing trips prior and during the pandemic. The goal was to quantify changes in ride-hailing travel during the pandemic and compare these changes with overall traffic volume variations.

Our findings for Chicago are as follows. First, ride-hailing travel was significantly reduced during the pandemic. The decrease in ride-hailing trips was much larger than the decrease in overall traffic volumes. Second, travel distances were generally much longer for non-shared trips during the pandemic, though travel times were not necessarily longer because the overall traffic network congestion was lower. Third, inter-census-tract short trips were fewer while the distances of such trips increased. These findings seem to suggest that, pre-pandemic, travelers appeared to be more likely to use ride-hailing for shorter trips but such trips decreased during the pandemic.

Submitted: September 22, 2020 AEDT, Accepted: October 26, 2020 AEDT

This is an open-access article distributed under the terms of the Creative Commons Attribution 4.0 


\section{REFERENCES}

Buzzfeednews. 2020. "Uber and Lyft Have Suspended Uber Pool And Shared Rides Due to the Coronoavirus.” Buzzfeednews. 2020.

Du, Jianhe, and Hesham A. Rakha. 2020. "Preliminary Investigation of COVID-19 Impact on Transportation System Delay, Energy Consumption and Emission Levels.” Transport Findings. https://doi.org/10.32866/001c.14103. 